\title{
A Statistical-Mechanical Analysis of Coded CDMA with Regular LDPC Codes
}

\author{
Toshiyuki Tanaka \\ Dept. of Electro. \& Info. Eng., \\ Tokyo Metropolitan Univ. \\ 1-1 Minami-Osawa, Hachioji, \\ Tokyo, 192-0397 Japan \\ e-mail: tanaka@eei.metro-u.ac.jp
}

\section{INTRODUCTION}

Performance of LDPC-coded CDMA system is analyzed, using the replica method of statistical mechanics (which has been separately applied to the analysis of CDMA [1] and of LDPC codes [2]), in the infinite codelength and large system (i.e., infinite number of users) limits. Most existing studies of such systems consider the case of a finite number of users. Caire et al. [3] consider the same limits as the current study, but theirs is based on stripping and successive detection/decoding. Our analysis, on the other hand, gives the information-theoretic and decoding thresholds of the optimum joint detection/decoding scheme.

\section{ANALYSIS}

We consider the serially concatenated system of a bank of per-user LDPC encoders and the standard fully-synchronous, randomly-spread, BPSK CDMA channel. We assume that the users are of equal rate, that the LDPC codes used are drawn from the same random ensemble of a regular $(C, L)$ LDPC code ( $C$ and $L$ are the column and row weights of the parity-check matrix), that the power control is perfect, and that the channel noise is additive white Gaussian with variance $\sigma_{0}^{2}$. The codelength $M$ and the number of users $K$ are sent to infinity, while the information rate $R \equiv N / M$ and the system load $\beta \equiv K / G$ are both kept to be $O(1)$, where $N$ and $G$ are the information-block length and the spreading factor, respectively.

Analytical evaluation of the performance of the optimum joint detection/decoding scheme requires solving the saddlepoint equations for $\{m, q, E, F, \pi(x), \hat{\pi}(\hat{x})\}$ :

$$
\begin{gathered}
m=\int \tanh \left(\sqrt{F} z+E+\sum_{c=1}^{C} \tanh ^{-1} \hat{x}_{c}\right) D z \mathcal{D}_{\hat{\pi}}^{C} \hat{x} \\
q=\int \tanh ^{2}\left(\sqrt{F} z+E+\sum_{c=1}^{C} \tanh ^{-1} \hat{x}_{c}\right) D z \mathcal{D}_{\hat{\pi}}^{C} \hat{x} \\
E=\frac{1}{\sigma^{2}+\beta(1-q)}, \quad F=\frac{\sigma_{0}^{2}+\beta(1-2 m+q)}{\left[\sigma^{2}+\beta(1-q)\right]^{2}} \\
\pi(x)=\int \delta\left[x-\tanh \left(\sqrt{F} z+E+\sum_{c=1}^{C-1} \tanh ^{-1} \hat{x}_{c}\right)\right] D z \mathcal{D}_{\hat{\pi}}^{C-1} \hat{x} \\
\hat{\pi}(\hat{x})=\int \delta\left(\hat{x}-\prod_{i=1}^{L-1} x_{i}\right) \mathcal{D}_{\pi}^{L-1} x
\end{gathered}
$$

where $D z \equiv(2 \pi)^{-1 / 2} e^{-z^{2} / 2} d z$ and $\mathcal{D}_{\hat{\pi}}^{C} \hat{\boldsymbol{x}} \equiv \prod_{c=1}^{C}\left[\hat{\pi}\left(\hat{x}_{c}\right) d \hat{x}_{c}\right]$ etc. The bit error rate $P_{b}$ (which is the same for all users since

\footnotetext{
${ }^{1}$ Support from EPSRC research grant GR/N00562 (TT, DS), and from Grant-in-aid for Scientific Reaearch on Priority Areas 14084209, MEXT, Japan, and from Bilateral Program between the UK and Japan, JSPS, Japan, (TT) is acknowledged.
}

\author{
David Saad ${ }^{1}$ \\ Neural Computing Res. Grp., \\ Aston Univ. \\ Aston Triangle, Birmingham, \\ B4 7ET United Kingdom \\ e-mail: d.saadQaston.ac.uk
}

they are statistically equivalent) is given by $P_{b}=\int_{-\infty}^{0} P(u) d u$ where

$P(u)=\int \delta\left[u-\tanh \left(\sqrt{F} z+E+\sum_{c=1}^{C} \tanh ^{-1} \hat{x}_{c}\right)\right] D z \hat{\mathcal{D}}^{C} \hat{\boldsymbol{x}}$

When the saddle-point equations have multiple solutions, the one minimizing the associated free energy (not shown) is information-theoretically relevant. The other solutions are also significant because they may determine the decoding threshold. Analytically found are the "error-free" solution $(m=q=1, \pi(x)=\delta(x-1), \hat{\pi}(\hat{x})=\delta(\hat{x}-1))$, and, in the limit $L \rightarrow \infty$, the one corresponding to the uncoded system [1]. Any other solutions are obtained numerically.

\section{RESULTS}

Figure 1 (a) shows the thresholds (decoding and information-theoretic), in terms of the critical load $\beta$, versus the noise level $\sigma^{2}$ for the joint detection/decoding scheme with $(3,6)$ - and $(4,8)$-LDPC codes. The maximum achievable spectral efficiency $\rho=\beta f$ ( $f$ being the free energy or mutual information per symbol and user) versus signal-to-noise ratio $E_{b} / N_{0}$ is shown in Fig. 1 (b), which shows that, although the spectral efficiency of the joint detection/decoding scheme practically converges to a finite value, it diverges theoretically, approaching that of the AWGN single-user spectral efficiency (shown as dotted line), as $\beta \rightarrow \infty$, suggesting possible improvement in the decoding threshold by adopting irregular LDPC codes.

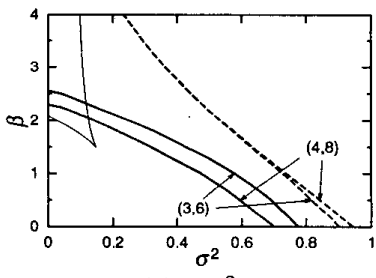

(a) $\beta-\sigma^{2}$

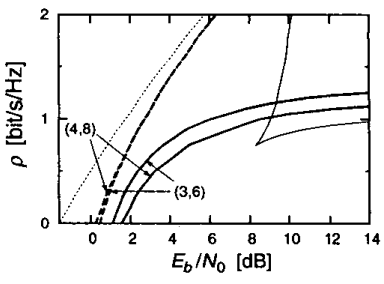

(b) $\rho-E_{b} / N_{0}$
Fig. 1: Decoding (thick) and information-theoretic (dashed) thresholds for $(3,6)$ - and $(4,8)$-LDPC coded CDMA systems.

\section{REFERENCES}

[1] T. Tanaka, "A statistical-mechanics approach to large-system analysis of CDMA multiuser detectors," IEEE Trans. Inf. Theory, vol. 48, pp. 2888-2910, 2002.

[2] T. Murayama, Y. Kabashima, D. Saad, and R. Vicente, "Statistical physics of rebgular low-density parity-check errorcorrecting codes," Phys. Rev. E, vol. 62, pp. 1577-1591, 2000.

[3] G. Caire, S. Guemghar, A. Roumy, and S. Verdú, "Maximizing the spectral efficiency of LDPC-encoded CDMA," submitted to IEEE Trans. Inf. Theory, 2002. 